\title{
Integrated analysis of gut microbiome and host immune responses in COVID-19
}

\author{
Xiaoguang $\mathrm{Xu}^{1, *}$, Wei Zhang ${ }^{1,2, *}$, Mingquan $\mathrm{Guo}^{3, *}$, Chenlu Xiao, ${ }^{4, *}$, Ziyu Fu ${ }^{1}$, Shuting $\mathrm{Yu}^{1}$, Lu Jiang ${ }^{1}$, Shengyue Wang ${ }^{1}$, \\ Yun Ling ${ }^{3}$, Feng Liu ${ }^{1}$, Yun Tan $(\bowtie)^{1}$, Saijuan Chen $(\bowtie)^{1}$ \\ ${ }^{1}$ Shanghai Institute of Hematology, State Key Laboratory of Medical Genomics, National Research Center for Translational Medicine at \\ Shanghai, Ruijin Hospital Affiliated to Shanghai Jiao Tong University School of Medicine, Shanghai 200025, China; ${ }^{2}$ School of Life Sciences \\ and Biotechnology, Shanghai Jiao Tong University, Shanghai 200240, China; ${ }^{3}$ Shanghai Public Health Clinical Center, Shanghai 201508, \\ China; ${ }^{4}$ Department of Laboratory Medicine, Ruijin Hospital Affiliated to Shanghai Jiao Tong University School of Medicine, Shanghai \\ 200025, China \\ (C) Higher Education Press 2022
}

\begin{abstract}
Emerging evidence indicates that the gut microbiome contributes to the host immune response to infectious diseases. Here, to explore the role of the gut microbiome in the host immune responses in COVID-19, we conducted shotgun metagenomic sequencing and immune profiling of 14 severe/critical and $24 \mathrm{mild} / \mathrm{moderate}$ COVID-19 cases as well as 31 healthy control samples. We found that the diversity of the gut microbiome was reduced in severe/critical COVID-19 cases compared to mild/moderate ones. We identified the abundance of some gut microbes altered post-SARS-CoV-2 infection and related to disease severity, such as Enterococcus faecium, Coprococcus comes, Roseburia intestinalis, Akkermansia muciniphila, Bacteroides cellulosilyticus and Blautia obeum. We further analyzed the correlation between the abundance of gut microbes and host responses, and obtained a correlation map between clinical features of COVID-19 and 16 severity-related gut microbe, including Coprococcus comes that was positively correlated with $\mathrm{CD3}^{+} / \mathrm{CD4}^{+} / \mathrm{CD8}^{+}$lymphocyte counts. In addition, an integrative analysis of gut microbiome and the transcriptome of peripheral blood mononuclear cells (PBMCs) showed that genes related to viral transcription and apoptosis were up-regulated in Coprococcus comes low samples. Moreover, a number of metabolic pathways in gut microbes were also found to be differentially enriched in severe/critical or mild/moderate COVID-19 cases, including the superpathways of polyamine biosynthesis II and sulfur oxidation that were suppressed in severe/critical COVID-19. Together, our study highlighted a potential regulatory role of severity related gut microbes in the immune response of host.
\end{abstract}

Keywords COVID-19; SARS-COV-2; gut microbiome; immune response

\section{Introduction}

The coronavirus disease 2019 (COVID-19), caused by the severe acute respiratory syndrome coronavirus 2 (SARSCoV-2), has become a pandemic threat to human health and the world economy. Although most cases of COVID19 are mild or moderate, $3 \%-4 \%$ of patients can be severe or critical, resulting in hospitalization, respiratory failure, or death [1-3]. Thus, unveiling disease severity-related

Received December 17, 2021; accepted December 31, 2021

Correspondence: Saijuan Chen, sjchen@stn.sh.cn; Yun Tan, ty12260@rjh.com.cn

*Xiaoguang Xu, Wei Zhang, Mingquan Guo, and Chenlu Xiao contributed equally to this study. factors is essential for the prognosis of COVID-19 and for exploring effective therapeutic approaches. Currently, studies focusing on host immune response have identified many features in severe/critical COVID-19 cases, such as cytokine storm, insufficient T cells response, lymphocytopenia, and aberrant activation of macrophage/monocytes [4-6]. However, the interplay between host and microbiome during the progression of COVID-19 remains unclear.

The gut intestine is the largest lymphoid organ in the human body and the primary source of memory T cells [7]. Recent studies have suggested that gut microbes play vital roles in regulating and training the host immune system, and are involved in multiple diseases, especially infectious diseases $[8,9]$. For example, gut microbes can interplay with the host immune system through direct physical 
contact, metabolites, and changes in the structural components of the intestinal mucosa [10,11]. Gut microbes can also engage in anti-viral response at the early stage of viral infection, and gut microbial depletion by antibiotics may suppress the anti-viral responses toward the influenza virus infection, leading to more severe symptoms [12]. In addition, genes related to cytokines, such as type I interferons, in the mononuclear phagocytes are suppressed in germ-free mice [13].

Recent studies have also noticed distinct changes in the gut microbiome post-SARS-CoV-2 infection. Compared to the non-infected cases, the composition of microbiome in COVID-19 seemed less diverse, even at six months after recovery $[14,15]$. Some opportunistic pathogens (e.g., Clostridium hathewayi and Bacteroides nordii) were increased in gut post-SARS-CoV-2 infection, while the abundance of some commensal symbionts (e.g., Eubacterium and Faecalibacterium) was decreased [16]. Additionally, several studies showed the gut microbiome was also related to the disease severity $[14,17]$. The abundance of some opportunistic pathogens (such as Corynebacterium, Enterococcus, and Enterobacter) appeared to be increased in severe cases of COVID-19 [17]. However, the interplay between the gut microbiota and host immune response and its impact on disease severity remains unclear.

Here, we profiled the gut microbiome of 14 severe/ critical and 24 mild/moderate COVID-19 cases and analyzed gut microbes' diversity, composition, and features between the two groups. These data were further compared to a group of 31 normal controls. Moreover, we investigated the correlation between the gut microbes and the clinical features (including serology, immune cell composition, and markers related to organ damage) and uncovered potential links between the gut microbe diversity and host immune response post-SARS-CoV-2 infection.

\section{Materials and methods}

\section{Enrollment}

Fourteen severe/critical and 24 mild/moderate COVID-19 cases were enrolled in this study. The COVID-19 was diagnosed and classified according to the Diagnosis and Treatment Protocol for COVID-19 Patients (Tentative 8th Edition).

\section{Fecal DNA extraction and shotgun metagenomic analysis}

Fecal samples were collected before any therapeutic treatment (such as antibiotics) after hospitalization and froze immediately with liquid nitrogen and stored in $-80{ }^{\circ} \mathrm{C}$ condition before DNA extraction. Fecal DNA was extracted according to the manufacture's instruction of the QIAamp Fast DNA Stool Mini Kit (Qiagen, German). Approximately $200 \mathrm{mg}$ of stool sample was used for DNA extraction.

500 ng DNA of each sample was used for library construction. The DNA libraries were prepared according to the manufacture's instruction of the KAPA Hyper Prep Kit. In brief, microbiome DNA was fragmented and tagged with adapters in one step and then added with index sequences during the PCR amplification. Libraries which passed the quality control analysis, including the Agilent 4200 analysis and quantifications, were used for sequencing. Libraries were sequenced on the NovaSeq 6000 platform (Illumina) using the $150 \mathrm{bp}$ paired-end strategy. A total of 31 healthy control samples, collected from the Sequence Read Archive database under the accession (SRP118759), were also used for analysis.

\section{Metagenomic analysis}

All sequenced reads were quality controlled by Trimmomatic (v0.39) [18] with the options "SLIDINGWINDOW:4:20" and "MINLEN:90." Clean reads were aligned to the human reference genome by bowtie2 (v2.4.4) [19] to remove human (host) derived reads. Approximately 44 million non-human reads on average ( 12 GB data) for each sample were reserved for subsequent analysis (Table S1). Taxonomic classification of bacterial and viral communities was inferred using kraken2 (v2.1.2) [20] and bracken (v2.5). Species profiles with read count were normalized to the Relative Log Expression (RLE) using the edgeR algorithm [21]. The alpha diversity was analyzed by the QIIME2 software [22]. The linear discriminant analysis effect size (LEfSe) [23] method was applied to identify taxa differentially abundant among mild/moderate, severe/critical COVID-19 patients, and healthy controls.

\section{Correlation between gut microbes and clinical features of host}

To determine the correlation between gut microbiota taxonomies and clinical biomarkers, the Pearson correlation coefficient was calculated using rcorr function in Hmisc package. The RNA-seq data sets of PMBCs from COVID-19 patients were obtained and processed as previously described [5]. Paired PBMC RNA-seq and gut microbiome samples from the same patient were used for analysis. Samples were divided into groups according to the abundance of Coprococcus comes or Enterococcus faecium, and the differentially expressed genes were obtained by the DESeq2 [24] analysis. Gene ontology analyses were conducted using the differentially expressed genes by topGO package. 


\section{Identification of differential metabolic pathways}

Metabolic pathways were inferred using HUMAnN3 [25] with default parameters. The functional profiles were normalized to copies per million (cpm), and the differential pathway analysis was performed using Student's $t$-test. All statistics were performed under the environment of $\mathrm{R}$ v4.0.2.

\section{Data availability}

The metagenomic sequencing data has been deposited in the NCBI Sequence Read Archive database under the accession number PRJNA792726.

\section{Results}

\section{Alpha diversity of gut microbes is decreased in severe/ critical COVID-19}

To investigate the relationship between the gut microbiome and COVID-19, we conducted shotgun metagenomic analysis of stool samples of 38 COVID-19 cases (14 severe/critical and $24 \mathrm{mild} /$ moderate) and 31 healthy controls. To avoid potential biases, the enrolled patients included basically even distributions of age, gender, and comorbidities (Table 1). Abundance of the gut microbes of each sample was illustrated in Table S2. PCA analysis using the abundance of the gut microbes in the 69 samples showed a continuum along with the severe/critical, mild/ moderate and healthy control groups (Fig. 1A), suggesting a stratification of the infection status and disease severity by the gut microbiome. Next, we analyzed the alpha diversity of gut microbes in each sample. We found that, compared to mild/moderate COVID-19 cases, the alpha diversity was significantly decreased in severe/critical cases (Fig. 1B). Finally, we visualized the composition of the gut microbes at the phylum level (Fig. 1C) and found that the composition notably differed between samples. Nevertheless, a relatively higher enrichment of Actinobacteria was observed in all COVID-19 patients, while a relatively higher enrichment of Firmicutes was observed in mild/moderate COVID-19 cases.

\section{Identification of gut microbes differentially associated with the severity of COVID-19}

To further examine the difference of gut microbiome among healthy control, mild/moderate, and severe/critical COVID-19 groups, we conducted a linear discriminant analysis (LDA) at the genus level (Fig. 2A-2C). Many differential gut microbes were identified among these three groups. Roseburia, Klebsiella, Coprococcus, and Dialister were decreased in mild/moderate COVID-19 cases compared to healthy control and this decrement was significantly deeper in severe/critical ones. Enterococcus

Table 1 Clinical features of the enrolled COVID-19 cases

\begin{tabular}{|c|c|c|c|}
\hline & $\begin{array}{l}\text { Severe/critical COVID-19 } \\
(n=14)\end{array}$ & $\begin{array}{l}\text { Mild/moderate COVID-19 } \\
(n=24)\end{array}$ & $P$ value \\
\hline Age (year) & $60.5(49.5-70.5)$ & $51.0(42.75-56.25)$ & $0.19^{\mathrm{a}}$ \\
\hline Gender & & & $1.00^{\mathrm{b}}$ \\
\hline Female $(n(\%))$ & $4(28.6 \%)$ & $7(29.2 \%)$ & \\
\hline Male $(n(\%))$ & $10(71.4 \%)$ & $17(70.8 \%)$ & \\
\hline Leukocyte counts $\left(\times 10^{9} / \mathrm{L}\right.$, normal range $\left.3.5-9.5\right)$ & $3.21(2.23-5.00)$ & $4.86(3.07-6.40)$ & $0.87^{\mathrm{a}}$ \\
\hline Lymphocytes $\left(\times 10^{9} / \mu \mathrm{L}\right.$, normal range $\left.1.1-3.2\right)$ & $0.87(0.64-1.15)$ & $1.86(1.47-2.25)$ & $<0.001^{\mathrm{a}}$ \\
\hline $\mathrm{CD}^{+} \mathrm{T}$ cell counts $(/ \mu \mathrm{L}$, normal range $690-2540)$ & $420(249-546)$ & $1204(1089.5-1610.5)$ & $<0.001^{\mathrm{a}}$ \\
\hline $\mathrm{CD}^{+} \mathrm{T}$ cell counts $(/ \mu \mathrm{L}$, normal range $190-1140)$ & $240(129.5-479.5)$ & $680(583.5-971.5)$ & $<0.001^{\mathrm{a}}$ \\
\hline $\mathrm{CD}^{+} \mathrm{T}$ cell counts $(/ \mu \mathrm{L}$, normal range $410-1590)$ & $143(100-192.5)$ & $380.5(242-594)$ & $<0.001^{\mathrm{a}}$ \\
\hline Platelets $\left(\times 10^{9} / \mathrm{L}\right.$, normal range $\left.125-350\right)$ & $147(133-200)$ & $248.5(225.3-277.8)$ & $<0.001^{\mathrm{a}}$ \\
\hline Hemoglobin (g/L, normal range $115-150)$ & $141(118-145)$ & $152(137-154.3)$ & $0.03^{\mathrm{a}}$ \\
\hline D-dimer $(\mu \mathrm{g} / \mathrm{L}$, normal range $0-0.5)$ & $0.44(0.38-0.81)$ & $0.22(0.18-0.28)$ & $0.04^{\mathrm{a}}$ \\
\hline \multicolumn{4}{|l|}{ Any comorbidities } \\
\hline Hypertension (n (\%)) & $4(28.6 \%)$ & $5(20.8 \%)$ & $0.70^{\mathrm{b}}$ \\
\hline Diabetes $(n(\%))$ & $2(14.3 \%)$ & $3(12.5 \%)$ & $1.00^{\mathrm{b}}$ \\
\hline Coronary heart disease $(n(\%))$ & $1(7.1 \%)$ & $1(4.2 \%)$ & $1.00^{\mathrm{b}}$ \\
\hline Chronic hepatitis B $(n(\%))$ & $1(7.1 \%)$ & $1(4.2 \%)$ & $1.00^{\mathrm{b}}$ \\
\hline Chronic renal diseases $(n(\%))$ & $1(7.1 \%)$ & $0(0.0 \%)$ & $0.37^{\mathrm{b}}$ \\
\hline
\end{tabular}

Data are presented as median (IQR).

Two-sided Mann-Whitney U test.

${ }^{\mathrm{b}}$ Fisher exact test. 
A

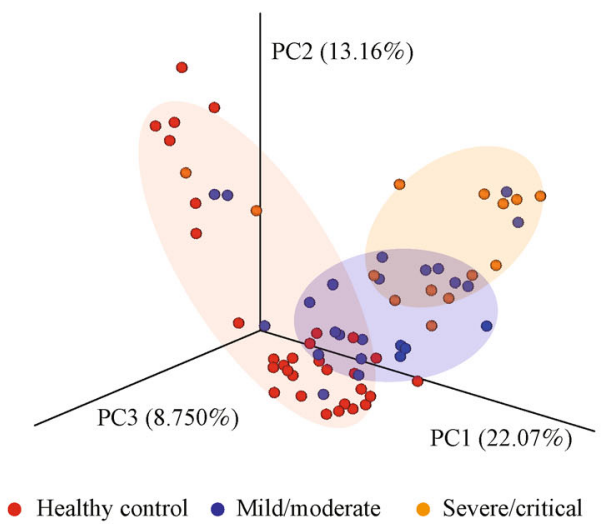

C
B



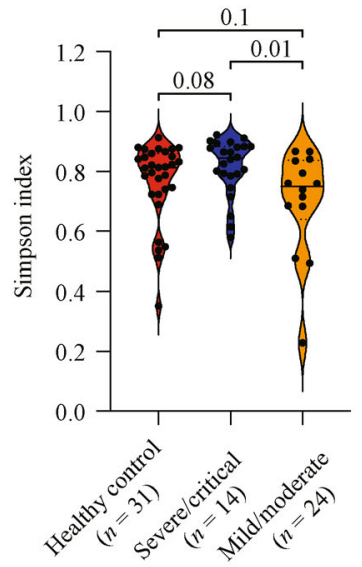

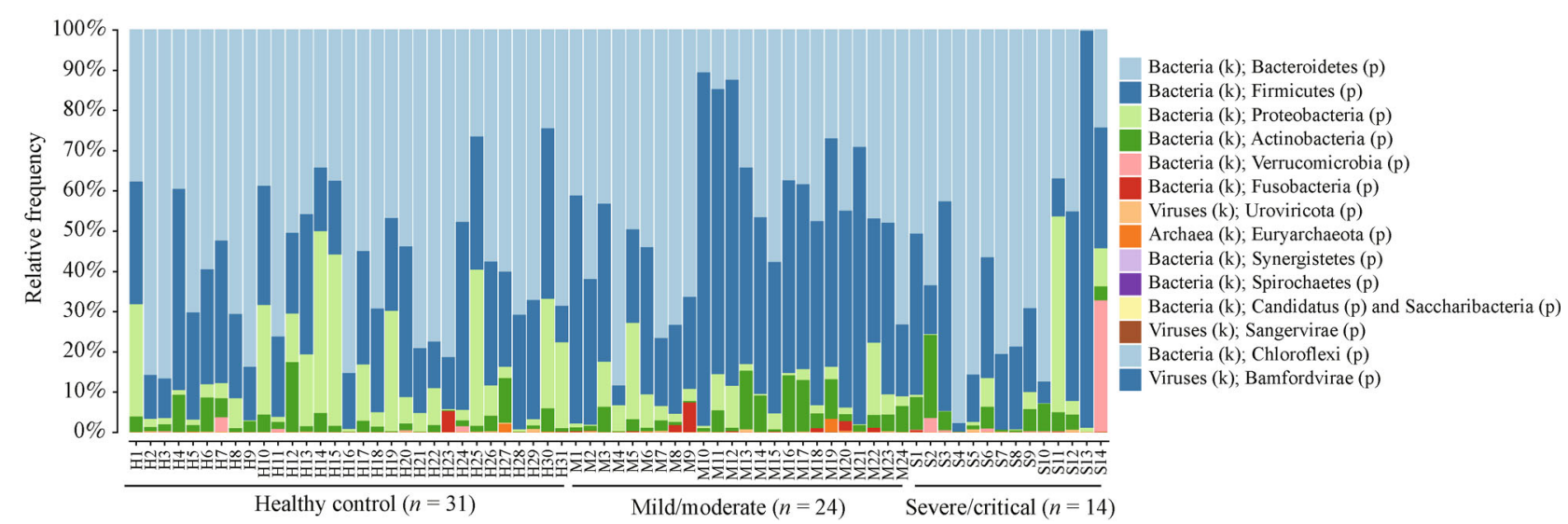

Fig. 1 Severe/critical COVID-19 showed less diversity of gut microbes. (A) PCA analysis shows the differences of composition of the gut microbes in severe/critical, mild/moderate COVID-19 and healthy controls. The abundance of gut microbes in each sample is used for PCA analysis. The red, blue and orange circles represent healthy controls, mild/moderate samples and severe/critical samples, respectively. (B) The severe/critical COVID-19 group shows less gut microbe diversity compared to the mild/moderate COVID-19 one. The gut microbe diversity per sample (alpha diversity) is calculated by the Shannon or Simpson method. Higher scores represent higher diversity of the gut microbes. (C) Illustration of the most abundant gut microbes at the phylum level in each sample. k, kingdom; p, phylum.

and Enterocloster were augmented in mild/moderate COVID-19 compared to healthy control and both were further increased in severe/critical cases. Mediterraneibacter, Blautia, Streptococcus, Anaerostipes and Anaerobutyricum were significantly increased in mild/moderate COVID-19 but their increase was to a lesser degree in severe/critical COVID-19 cases.

Next, we performed the same analysis at the species level, and identified 16 species enriched in healthy control, mild/moderate, or severe/critical COVID-19 groups (Fig. 2D). Enterococcus faecium, Enterococcus avium, and Enterococcus durans were significantly increased in mild/moderate COVID-19 and the increment was even more remarkable in severe/critical COVID-19. Lachnospira eligens was significantly decreased in mild/moderate COVID-19, while this species together with Klebsiella pneumoniae, and Roseburia intestinalis were significantly suppressed in severe/critical COVID-19. The abundance of these gut microbes thus might be consistently increased or decreased during the progression toward severe/critical COVID-19. On the other hand, Akkermansia muciniphila, Bacteroides ovatus, and Bacteroides cellulosilyticus were decreased or not changed in mild/moderate COVID-19 but tended to be increased in severe/critical COVID-19, whereas Streptococcus oralis, Coprococcus catus, Anaerostipes hadrus, Blautia obeum, and Coprococcus comes tended to increase in mild/moderate COVID-19 but remained basically unchanged in severe/critical COVID19. Ruminococcus gnavus and Ruminococcus torques differed from other species since they showed significant increase in mild/moderate COVID-19 while in severe/ critical cases, a lesser extent of increase and a significant decrease, compared to normal controls, were revealed, respectively. These observations suggested that some gut microbes might have undergone complex dynamical changes during the progression of COVID-19. 
A

Severe vs. mild

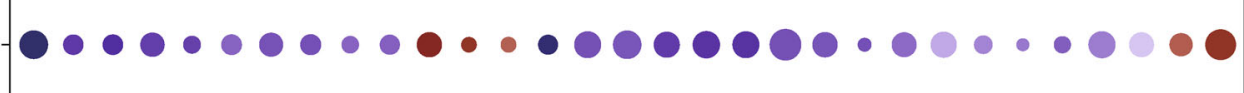

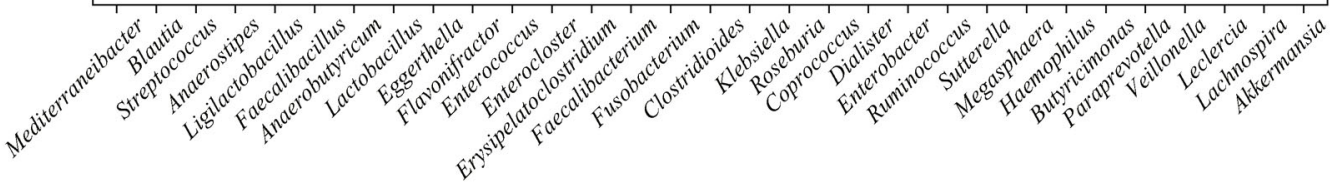

LDA

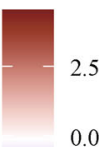

B

Severe vs. healthy
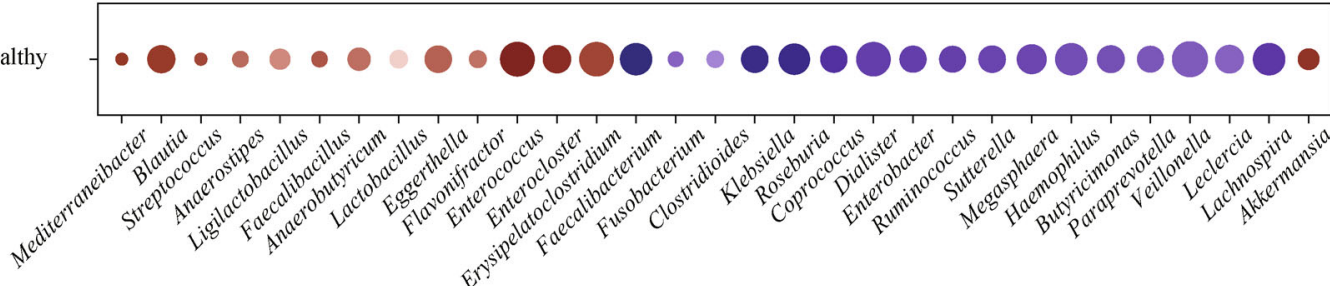

C

Mild vs. healthy
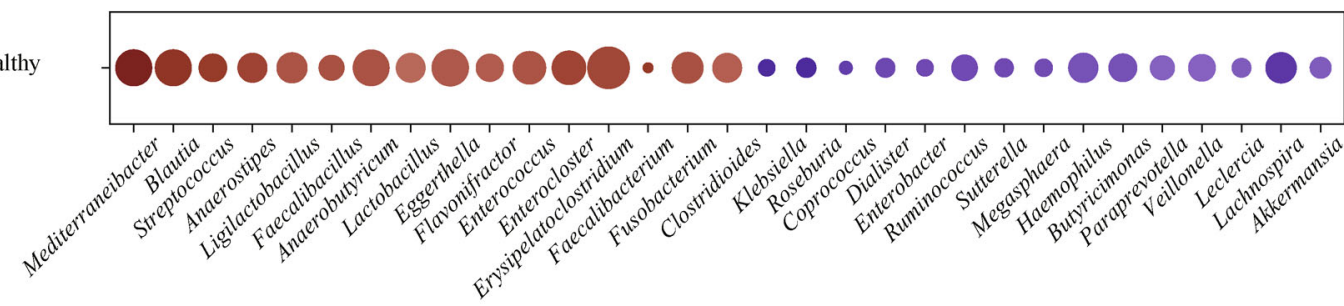

$\mathrm{D}$

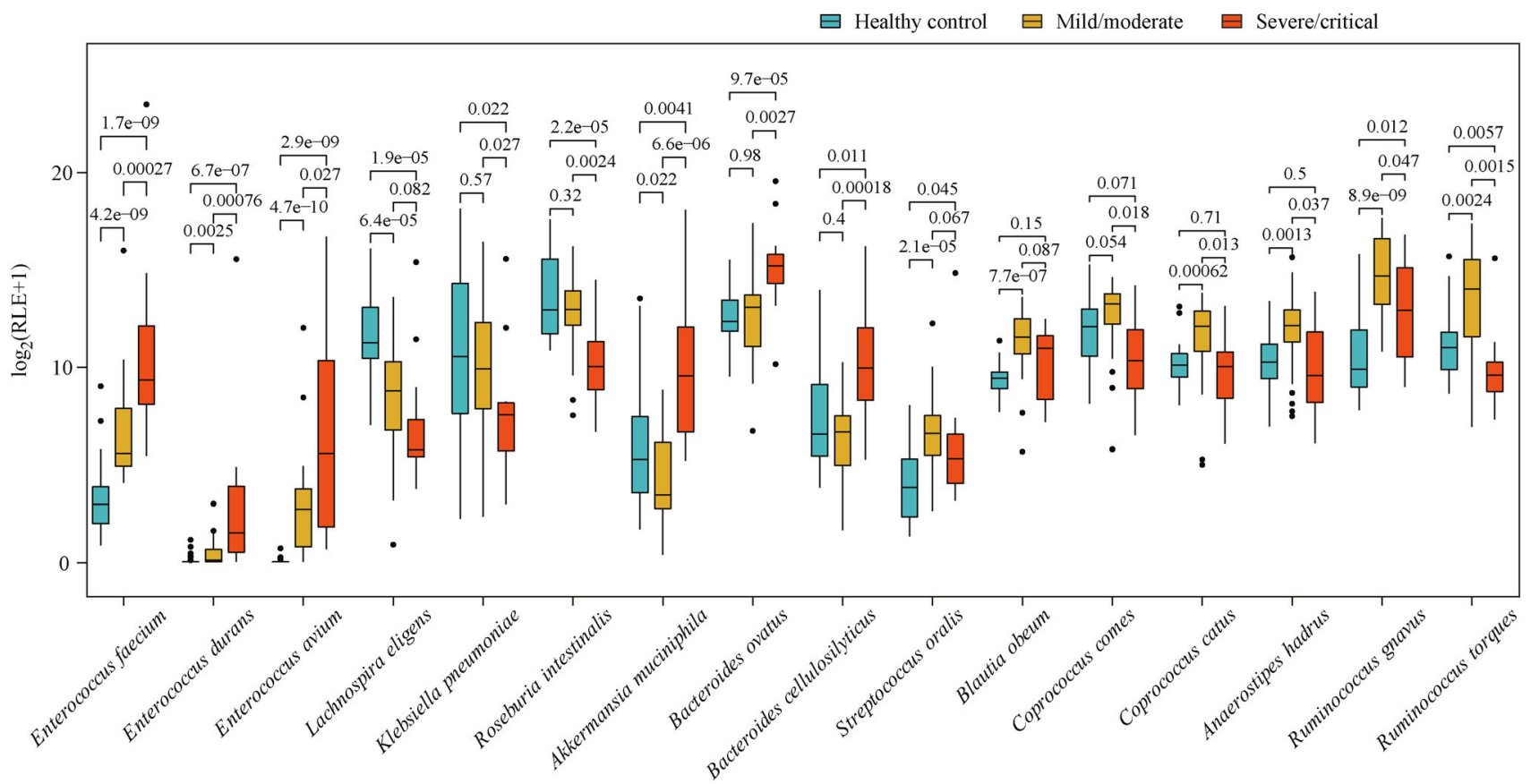

Fig. 2 Identification of severity-related gut microbes in COVID-19. (A) Feature gut microbe between severe/critical and mild/moderate COVID-19. (B) Feature gut microbe between severe/critical COVID-19 and health control. (C) Feature gut microbes between mild/moderate COVID-19 and health control. Gut microbes at genus levels and LDA (Linear discriminant analysis) $>3.0$ are plotted. LDA values are shown by colors, and $P$ values are shown by the sizes of the circles. (D) Illustration of top differential species between severe/critical and mild/moderate COVID-19 cases compared to healthy controls. 


\section{Correlations between the abundance of severity-related gut microbes and clinical features of COVID-19}

To investigate the relationship between the gut microbes and the clinical features of COVID-19, we analyzed correlations between gut microbes and immune cell populations and blood cell functions. We found that Roseburia intestinalis, Coprococcus catus, Coprococcus comes, and Blautia obeum were positively correlated with lymphocyte counts, lymphocyte percent, $\mathrm{CD}^{+}$cell counts, $\mathrm{CD}^{+}$cell counts, and $\mathrm{CD} 8^{+}$cell counts (Fig. $3 \mathrm{~A}$ and $3 \mathrm{~B}$, Table S3), indicating a possible auxiliary role of these bacteria in the lymphoid response to SARS-COV-2 infection. Second, we analyzed the correlation between gut microbes and markers of organ damage or dysfunction. Akkermansia muciniphila and Bacteroides cellulosilyticus were found to be positively correlated with the aspartate transaminase, creatine kinase isoenzymes, and lactate dehydrogenase, and Streptococcus oralis was positively correlated with gamma-glutamyltransferase (Fig. 3C and 3D), while Roseburia intestinalis was negatively correlated with direct bilirubin (Fig. 3C and 3D, Table S4), suggesting a potential link between gut microbes and potential organ damage in COVID-19. A few significant correlations were found between the gut microbes and the cytokines or coagulation parameters. For instance, Klebsiella pneumoniae was positively correlated to interferon gamma. Coprococcus comes was negatively correlated to the levels of fibrinogen, while Enterococcus durans and Enterococcus faecium were positively correlated to the levels of activated partial thromboplastin time (APTT) (Fig. 3E-3G, Tables S5 and S6).

\section{Potential impact of gut microbes on host immune responses}

To further explore the potential impact of the gut microbes on the host immune response, we conducted an integrative analysis using the abundance of gut microbes and the transcriptomic features of the PBMC samples of the same COVID-19 patients.

We selected Coprococcus comes and Enterococcus faecium for the analysis because (1) they were associated with multiple markers of immune response, (2) they were abundant in most samples. We divided COVID-19 samples into two groups each time based on the median abundance of Coprococcus comes and Enterococcus faecium, respectively. We then identified differentially expressed genes in PBMCs between these two groups. We found that many genes were upregulated in Coprococcus comes low group (Fig. 4A). Gene ontology showed that these upregulated genes were enriched in pathways related to viral transcription, regulation of signal transduction by $\mathrm{p} 53$, and positive regulation of intrinsic apoptotic signaling (Fig. 4B), indicating that a low level of Coprococcus comes might lead to viral transcription and apoptosis of PBMCs.

Also, a large number of genes in PBMCs were upregulated in Enterococcus faecium high group (Fig. 4C). Gene ontology analysis showed that these upregulated genes were enriched in pathways related to platelet aggregation, intrinsic blood coagulation, neutrophil degranulation, and defense response to Gram-negative bacterium (Fig. 4D). These data might indicate that a high level of Enterococcus faecium could be engaged in the aberrant innate immune response and coagulation.

\section{Identification of potential metabolic pathways affected by intestinal microbiome in COVID-19}

Furthermore, we explored if metabolic pathways might be altered by gut microbes in severe COVID-19 cases, since lines of evidence demonstrate gut microbes regulate the host immune response mainly via its metabolites. To that end, we computed the enrichment among 506 metabolic pathways of gut microbiomes and analyzed the difference of each pathway between the mild/moderate and severe/ critical COVID-19 groups (Table S7). Three pathways were highly enriched in the severe/critical group (e.g., lipid IVA biosynthesis (E. coli) and pyridoxal 5'-phosphate biosynthesis I), while 14 other pathways were highly enriched in the mild/moderate group (e.g., superpathways of glycerol degradation to 1,3-propanediol, sulfur oxidation, and polyamine biosynthesis II) (Fig. 5A and 5C). We further analyzed the species contributions in differential enriched pathways using HUMAnN3. The analysis suggested that the differential enrichment of the superpathway of glycerol degradation to 1,3-propanediol could be mainly attributed to Ruminococcus torques. The superpathway of sulfur oxidation was mainly attributed to Eubacterium rectale, Ruminococcus torques, and Blautia obeum, while the superpathway of polyamine biosynthesis could be mainly attributed to Escherichia coli (Fig. 5B). Meanwhile, the differential enrichment of the lipid IVA biosynthesis (E. coli) and pyridoxal 5'-phosphate biosynthesis I could primarily result from Bacteroides uniformis and Bacteroides stercoris (Fig. 5C).

\section{Discussion}

In this study, we carried out metagenomics study in a cohort of COVID-19, and identified distinct host features correlated to the abundance of the gut microbes. Consistent with previous studies $[14,17]$, we found that the abundance and composition of the gut microbiome in severe/critical cases were different from the mild/moderate ones. We used two methods to compute the alpha diversity, and the results supported the notion that the diversity of gut microbes was significantly reduced in severe/critical COVID-19 
A



B


E

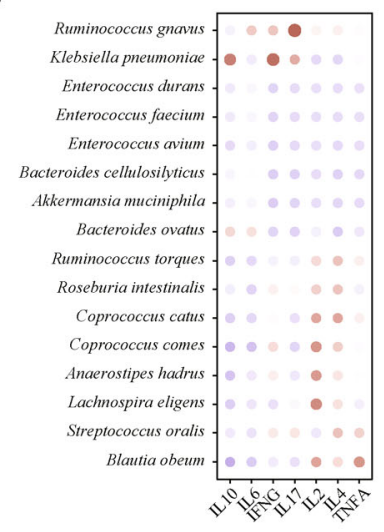

C

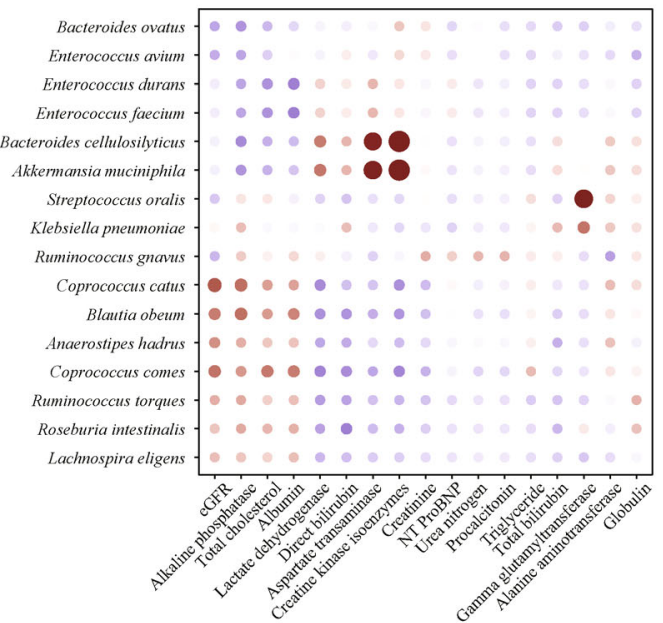

F

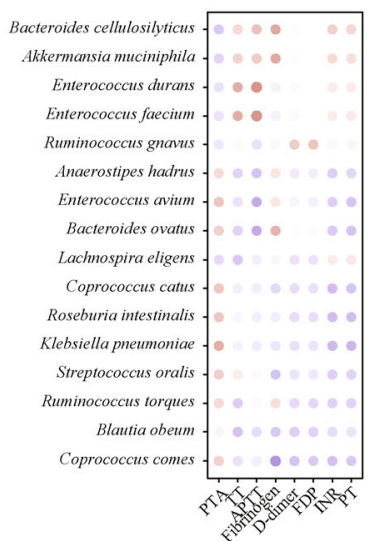

D
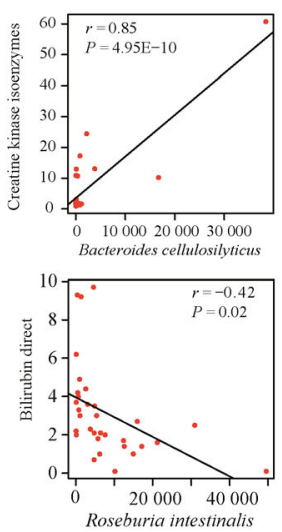

G
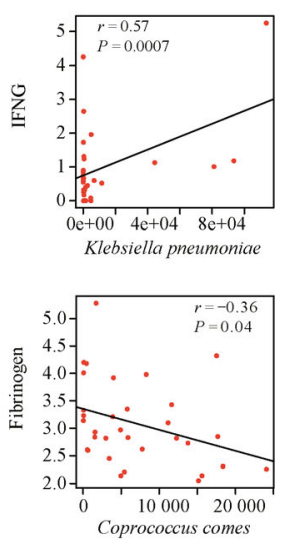

Fig. 3 Correlation between severity-related gut microbes and clinical features of COVID-19. (A and B) Analysis of the correlation between severityrelated gut microbes and the blood features. The Pearson correlation coefficient and $P$ value are used for plotting. Similar scores are clustered by the unsupervised clustering. Sixteen severity-related gut microbes and 34 blood features are used for plotting. Representative correlations are shown in panel B. (C and D) Analysis of the correlation between severity-related gut microbes and markers associated with organ damage or dysfunction. Representative correlations are shown in panel D. (E-G) Analysis of the correlation between severity-related gut microbes and cytokines or markers associated with coagulation. Representative correlations are shown in panel G. 
A

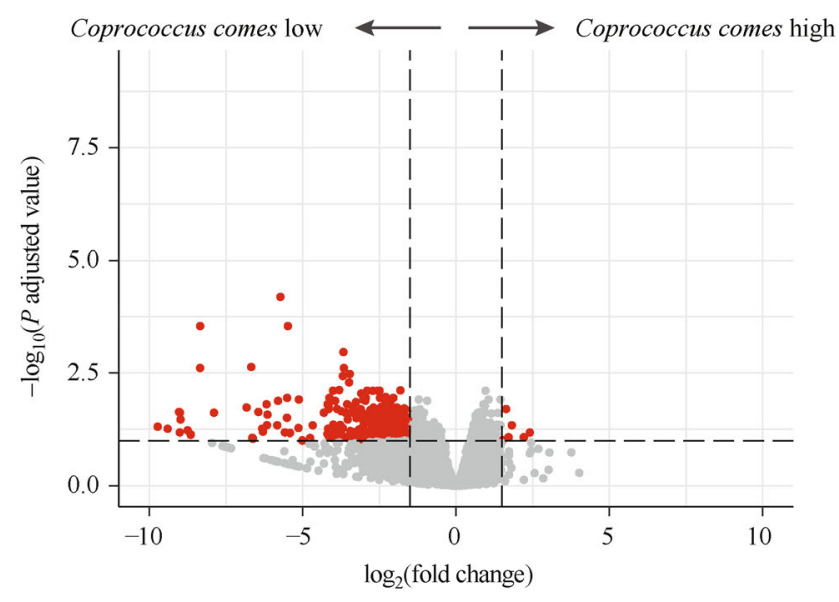

B

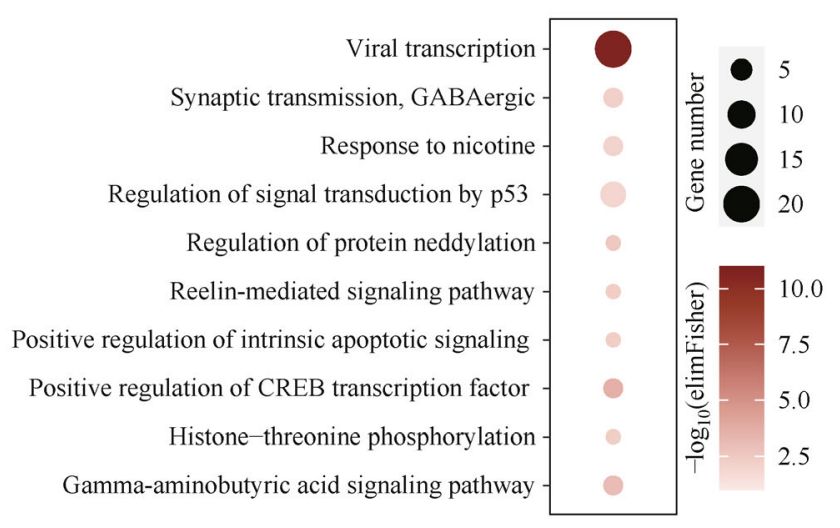

D

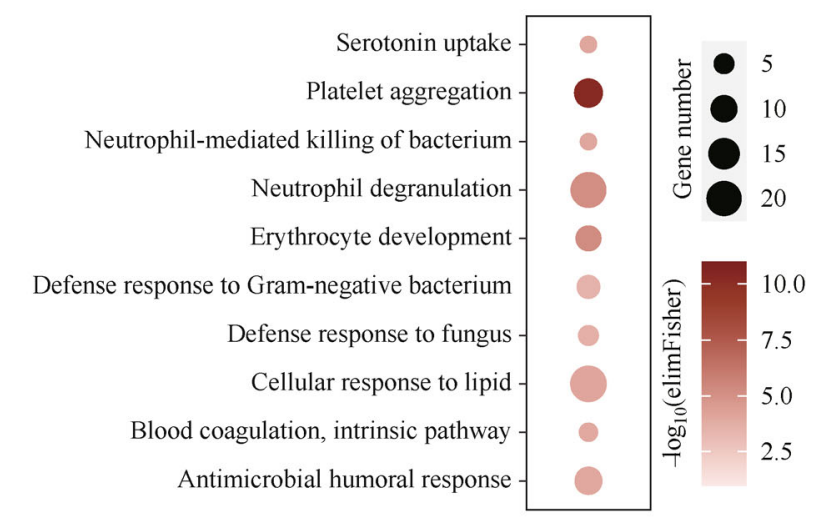

C

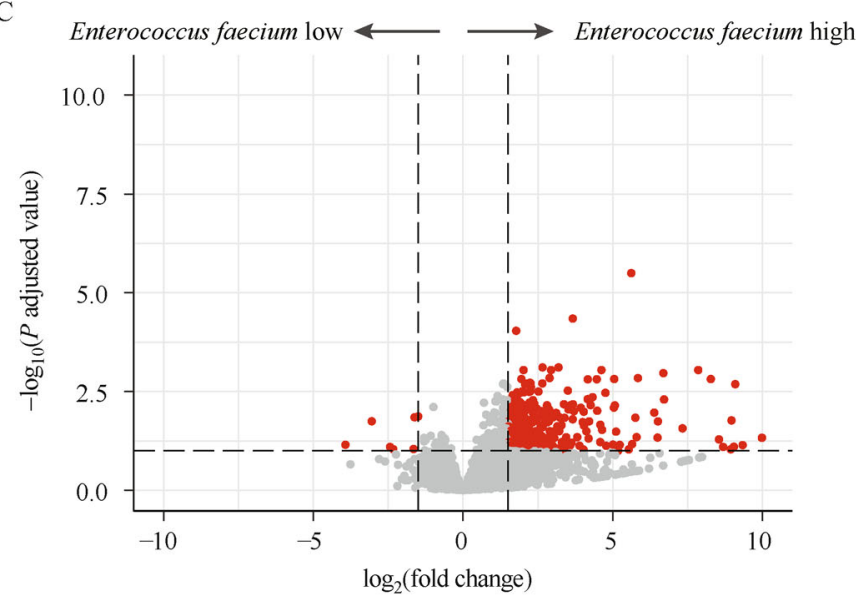

Fig. 4 Potential impact of severity-related gut microbes on the transcriptomic features of PBMCs in COVID-19. (A) Differentially expressed genes in PBMCs between Coprococcus comes high and low groups. Samples are divided into two groups based on the median abundance of the Coprococcus comes in gut microbes. Genes expressed at higher levels in Coprococcus comes high group (right) or Coprococcus comes low group (left) are shown in red. (B) Gene ontology analysis using genes upregulated in the Coprococcus comes low group. (C) Differentially expressed genes in PBMCs between Enterococcus faecium high and low groups. Samples are divided into two groups based on the median abundance of the Enterococcus faecium in gut microbes. Genes expressed at higher levels in Enterococcus faecium high group (right) or Enterococcus faecium low group (left) are shown in red. (D) Gene ontology analysis for genes upregulated in the Enterococcus faecium high group.

compared to the mild/moderate COVID-19 [16,17]. Nevertheless, the differences in gut microbiome diversity between COVID-19 and healthy control remain controversial $[14,26,27]$. Some reported no significant difference in the alpha diversity between healthy control and COVID$19[14,26]$, while others described a decrease of it in COVID-19 [27]. Here, we found no significant differences in alpha diversity between healthy control and either mild/ moderate or severe/critical COVID-19. These different results might be attributed to different ages, comorbidities or antibiotic usages among different cohorts.

Then we identified a number of differential gut microbes among normal controls, mild/moderate and severe/critical COVID-19 at the genus and species levels. Consistent with previous studies, we found Coprococcus and Roseburia were lower in COVID-19 compared to healthy controls [28], while Enterococcus was higher in severe/critical COVID-19 compared to the mild/moderate ones [16,17]. At the species level, we confirmed previous reports that the abundance of Akkermansia muciniphila and Enterococcus faecium were increased, while Roseburia intestinalis and Coprococcus comes were decreased, in severe COVID-19 compared to mild/moderate ones [14,16,29]. However, scrutiny of changes of species allowed us to uncover unique severity-associated patterns: some species were upor downregulated in a similar way in both mild/moderate and severe/critical cases compared to healthy control, such as Enterococcus faecium, Enterococcus durans, 
A

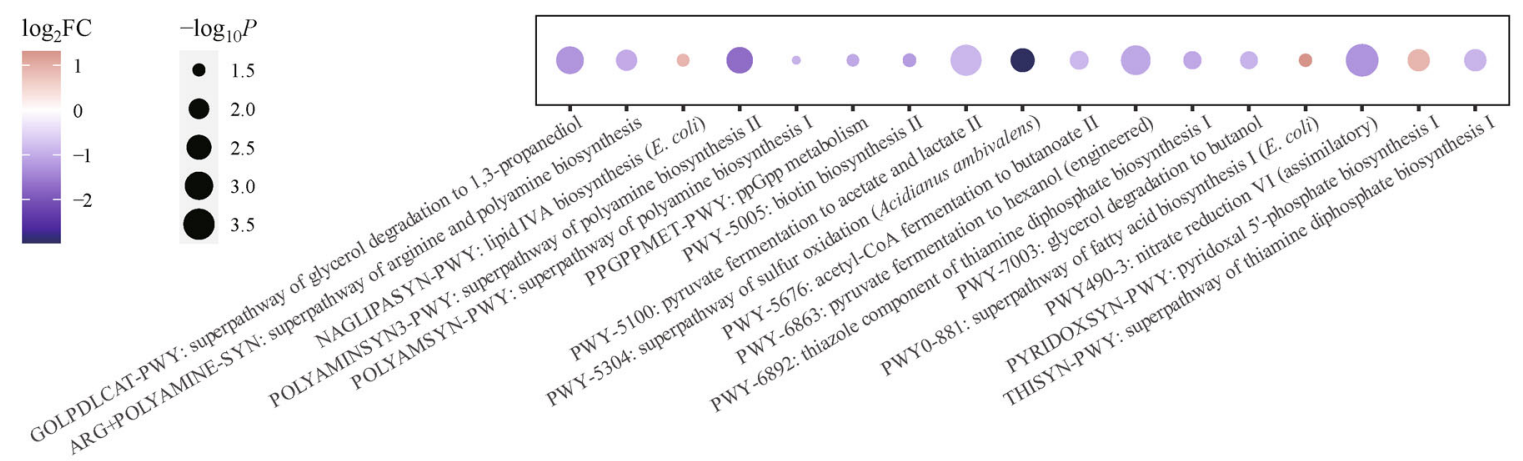

B

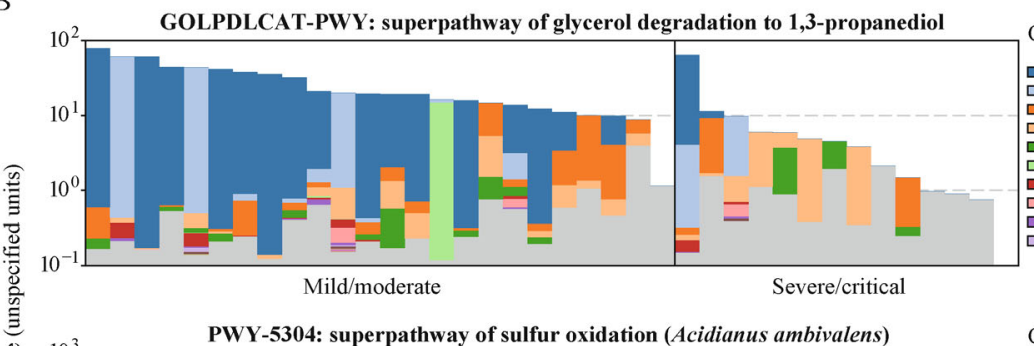

Contributions (linear scaling within total bar height):
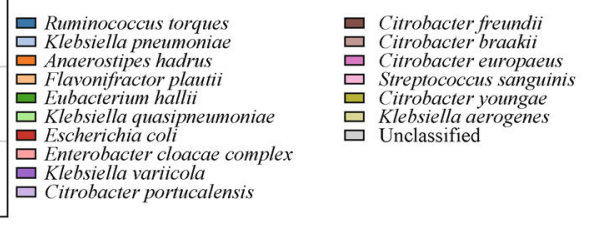

믈 Cirrobacter freundii

$\square$ Citrobacter europaeus

ㅁ. Citrobacter youngae Unclassified

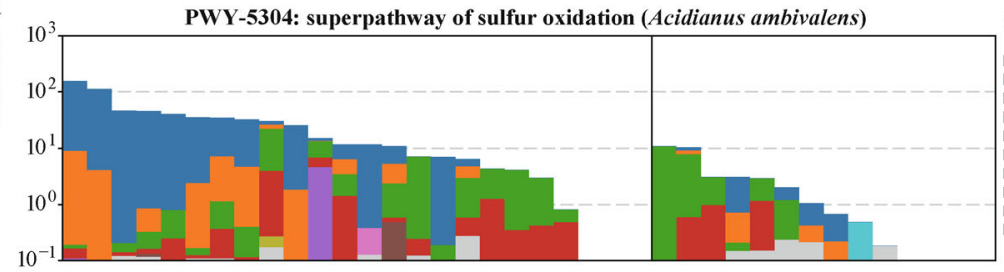

Contributions (linear scaling within total bar height): 口 Eubacterium rectale Blantia obeum Anaerostipes hadrus Coprococcus eutactus Megasphaera elsdenii Saccharomyces cerevisia Acinetobacter baumannii $10^{-1}$

Mild/moderate

Severe/critical

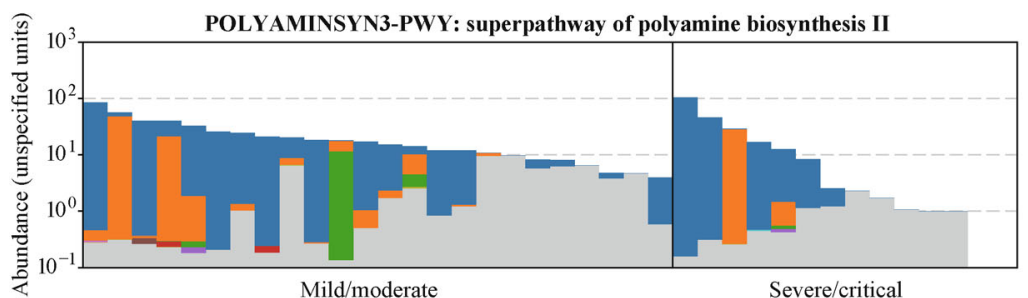

Contributions (linear scaling within total bar height): ㅁ Escherichia coli - Klebsiella pneumoniae - Clostridium saccharolyticum - Enterobacter cloacae complex Klebsiella aerogenes Escherichia fergusoni - Raoultella planticola Raoultella planticola
Mild $/$ moderate
Severe/critical

C

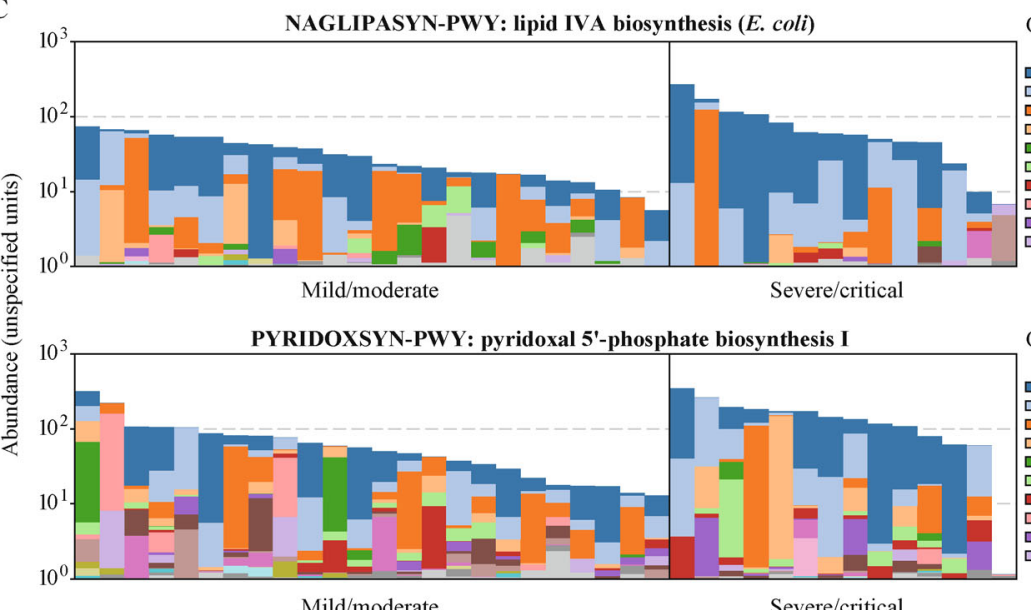

Contributions (linear scaling within total bar height):

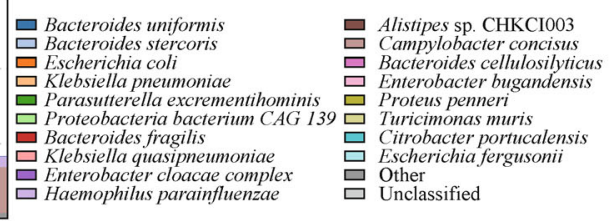

Contributions (linear scaling within total bar height):

$\square$ Bacteroides stercoris Bacteroides uniformis Escherichia coli Bacteroides thetaiotaomicron Parabacteroides distason Bacteroides ovatus Bacteroides dorei Bacteroides fragilis

Mild/moderate

Severe/critical

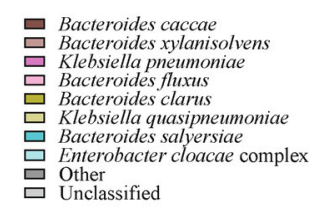

Fig. 5 Differentially enriched metabolic pathways between severe/critical and mild/moderate COVID-19. (A) Differentially enriched metabolic pathways between mild/moderate and severe/critical COVID-19. The enrichment of each sample in the 506 metabolic pathways is calculated, and the fold change and $P$ value of the enrichment score between the mild/moderate and severe/critical groups are analyzed. Top differentially enriched pathways are plotted. (B and C) Representative illustration of species contributions to the differential enriched KEGG metabolic pathway between mild/moderate and severe/critical COVID-19. The species contributions to DNA abundance of GOLPDLCAT-PWY, PWY-5304, POLYAMINSYN3PWY, NAGLIPASYN-PWY, and PYRIDOXSYN-PWY are plotted. 
Enterococcus avium, Lachnospira eligens, Klebsiella pneumoniae, and Roseburia intestinalis, and the degree of changes was much higher in severe/critical group than that in mild/moderate one. The changes of these species were thus likely induced by SARS-CoV-2 infection. By contrast, others showed modification patterns (up- or downregulation) in opposite direction in mild/moderate or severe/critical COVID-19 compared to healthy control, such as Coprococcus comes, Akkermansia muciniphila, and Ruminococcus torques. Understanding the biology behind the complex pattern of these species is a challenge.

Although an array of severity-related gut microbes in COVID-19 was established, how they could impact the host immune response remained obscure. We conducted an integrative study using the gut microbiome profile, clinical features, and the transcriptomic profile of PMBCs in the host, and revealed several links between gut microbes and host responses. Of note, Roseburia intestinalis, Coprococcus catus, Coprococcus comes, and Blautia obeum were positively correlated with lymphoid-related markers, such as lymphocyte counts, lymphocyte percent, $\mathrm{CD}^{+}$ cell counts, $\mathrm{CD}^{+}$cell counts, and $\mathrm{CD}^{+}$cell counts, providing evidence for a potential interplay between these gut microbes and lymphocyte regulation. These four gut microbes were either moderately reduced, remained unchanged or even moderately increased in mild/moderate COVID-19, whereas the former three of them were remarkably reduced in severe/critical cases among whom the lymphocytopenia is one of the hallmarks at the initial infectious stage $[4,30]$. It is tempting to speculate that the imbalance of these gut microbes might be a key for dysregulated lymphocytes response in severe/critical COVID-19. Indeed, previous studies have demonstrated the regulatory function in some of these gut microbes on lymphocyte differentiation or regulation [31-33]. For example, Roseburia intestinalis could suppress the production of interleukin-17 and promote $T$ cell differentiation [31], while Coprococcus comes was related with IL-1b and IL-6 during the stimulation of Candida albicans hyphae [32]. Therefore, the correlation among these gut microbes and lymphocytes might reflect the potential effect of gut microbes on the immune response toward SARS-CoV-2 infection.

Besides, we found Bacteroides cellulosilyticus and Akkermansia muciniphila were positively correlated with an increment of creatine kinase isoenzyme (markers related to potential cardiovascular impairment) and aspartate transaminase (markers related to potential liver injury), and Streptococcus oralis was positively correlated with gamma-glutamyltransferase (markers related to potential liver injury). In fact, the Bacteroides cellulosilyticus and Streptococcus oralis could be involved in liver dysfunction as higher levels of Bacteroides cellulosilyticus were observed in advanced fibrosis in nonalcoholic fatty liver disease [34], while higher levels of Streptococcus oralis were observed in cirrhosis compared to healthy subject [35]. Aberrant abundance of Bacteroides cellulosilyticus was also observed in atherosclerotic cardiovascular disease compared to healthy individuals [36]. The supplementation of live Akkermansia muciniphila in human volunteers could slightly increase the creatine kinase level [37], implying a potential regulatory function of Akkermansia muciniphila in cardiovascular disease. These observations indicated that the increased levels of gut microbes might contribute to multi-organ damage or dysfunction in severe/ critical COVID-19.

In addition to the laboratory test, we also analyzed the impact of Coprococcus comes and Enterococcus faecalis on the gene expression profile in PBMCs. The Coprococcus comes was found to be suppressed in severe/critical COVID-19, while this species might inhibit the expression of genes related to viral transcription and intrinsic apoptotic signaling. Moreover, the Enterococcus faecalis was shown to be enriched in severe/critical COVID-19, which was related to enhanced expression of genes related to platelet aggregation and neutrophil degranulation. Previous studies demonstrated that some clinical isolates of Enterococcus faecalis could cause aggregation of human platelets [38] or activate human platelets in vitro [39], while sonicated extract of Enterococcus faecalis could suppress the cell cycle transition and inhibit lymphocytes response [40]. Hence, we assume that the observed dysregulation of certain gut microbes might not be a passenger but a potential driver for the severity of COVID-19.

Finally, using pathway enrichment analysis of gut microbiome, we found several metabolic pathways differentially enriched between mild/moderate and severe/critical COVID-19. For instance, the enrichment of the superpathway of polyamine biosynthesis II was much lower in severe/critical compared to mild/moderate COVID-19. Lines of evidence have demonstrated the polyamine is involved in the immune response $[41,42]$ in that it is required for normal $\mathrm{T}$ lymphocyte proliferation while capable of inhibiting the production of proinflammatory cytokines such as IL1 $\beta$ and IL6 in mouse macrophages [41-44]. It is therefore reasonable to hypothesize that the changes of gut microbes in severe/ critical COVID-19 might contribute to suppressed polyamine biosynthesis, thereby leading to decreased $\mathrm{T}$ cell proliferation and aberrantly increased cytokine production. Future metabolomic studies in COVID-19 should be conducted to further elucidate the interplay among gut microbes, gut and body metabolites, and host immune response.

In summary, our study highlights the role of gut microbes in the potential regulation of disease severity of COVID-19, and provides evidence for the interplay between gut microbes and host response. Still, more efforts are warranted to understand the cellular and 
molecular mechanisms underlying the interplay. How gut microbes are engaged in the sequelae of the severe/critical COVID-19 should also be addressed, since it takes a long time for these patients to recover their microbiome.

\section{Acknowledgements}

This work was supported by grants from National Natural Science Foundation of China (Nos. 8210010124, 81890994, and 81861148030), Double First-Class Project (No. WF510162602) from the Ministry of Education, State Key Laboratory of Medical Genomics, Overseas Expertise Introduction Project for Discipline Innovation (111 Project, No. B17029), National Key R\&D Program of China (No. 2019YFA0905902), Natural Science Foundation of Shanghai (Nos. 21ZR1480900 and 21YF1427900), Shanghai Clinical Research Center for Hematologic Disease (No. 19MC1910700), Shanghai Major Project for Clinical Medicine (No. 2017ZZ01002), Shanghai Shenkang Hospital Development Center (No. SHDC2020CR5002), Innovative Research Team of High-level Local Universities in Shanghai, Shanghai Collaborative Innovation Program on Regenerative Medicine and Stem Cell Research (No. 2019CXJQ01), Shanghai Jiao Tong University (No. YG2021QN19), Shanghai Guangci Translational Medical Research Development Foundation. We thank the support from Prof. Hai Fang, the ASTRA computing platform, and the high-throughput sequencing platform in the National Research Center for Translational Medicine (Shanghai). In addition, we thank the Pi computing platform in the Center for High-Performance Computing at Shanghai Jiao Tong University.

\section{Compliance with ethics guidelines}

Xiaoguang Xu, Wei Zhang, Mingquan Guo, Chenlu Xiao, Ziyu Fu, Shuting Yu, Lu Jiang, Shengyue Wang, Yun Ling, Feng Liu, Yun Tan, and Saijuan Chen declared no potential conflicts of interest with respect to the research, authorship, and/or publication of this article. All procedures followed were in accordance with the ethical standards of the responsible committee on human experimentation (institutional and national) and with the Helsinki Declaration of 1975, as revised in 2000(5). Informed consent was obtained from all patients for being included in the study.

Electronic Supplementary Material Supplementary material is available in the online version of this article at https:/doi.org/ $10.1007 / \mathrm{s} 11684-022-0921-6$ and is accessible for authorized users.

\section{References}

1. Shen Y, Zheng F, Sun D, Ling Y, Chen J, Li F, Li T, Qian Z, Zhang Y, Xu Q, Liu L, Huang Q, Shan F, Xu L, Wu J, Zhu Z, Song Z, Li S, Shi Y, Zhang J, Wu X, Mendelsohn JB, Zhu T, Lu H. Epidemiology and clinical course of COVID-19 in Shanghai, China. Emerg Microbes Infect 2020; 9(1): 1537-1545

2. Taleghani N, Taghipour F. Diagnosis of COVID-19 for controlling the pandemic: a review of the state-of-the-art. Biosens Bioelectron 2021; 174: 112830

3. D'Arienzo M, Coniglio A. Assessment of the SARS-CoV-2 basic reproduction number, $R_{0}$, based on the early phase of COVID-19 outbreak in Italy. Biosaf Health 2020; 2(2): 57-59

4. Zhang X, Tan Y, Ling Y, Lu G, Liu F, Yi Z, Jia X, Wu M, Shi B, Xu S, Chen J, Wang W, Chen B, Jiang L, Yu S, Lu J, Wang J, Xu M, Yuan Z, Zhang Q, Zhang X, Zhao G, Wang S, Chen S, Lu H. Viral and host factors related to the clinical outcome of COVID-19. Nature 2020; 583(7816): 437-440

5. Tan Y, Zhang W, Zhu Z, Qiao N, Ling Y, Guo M, Yin T, Fang H, Xu X, Lu G, Zhang P, Yang S, Fu Z, Liang D, Xie Y, Zhang R, Jiang L, Yu S, Lu J, Jiang F, Chen J, Xiao C, Wang S, Chen S, Bian XW, $\mathrm{Lu} \mathrm{H}$, Liu F, Chen S. Integrating longitudinal clinical laboratory tests with targeted proteomic and transcriptomic analyses reveal the landscape of host responses in COVID-19. Cell Discov 2021; 7(1): 42

6. Jin A, Yan B, Hua W, Feng D, Xu B, Liang L, Guo C. Clinical characteristics of patients diagnosed with COVID-19 in Beijing. Biosaf Health 2020; 2(2): 104-111

7. Kok L, Masopust D, Schumacher TN. The precursors of CD8 ${ }^{+}$ tissue resident memory $\mathrm{T}$ cells: from lymphoid organs to infected tissues. Nat Rev Immunol 2021; [Epub ahead of print] doi: 10.1038/ s41577-021-00590-3

8. Krautkramer KA, Fan J, Bäckhed F. Gut microbial metabolites as multi-kingdom intermediates. Nat Rev Microbiol 2021; 19(2): 77-94

9. Fan Y, Pedersen O. Gut microbiota in human metabolic health and disease. Nat Rev Microbiol 2021; 19(1): 55-71

10. Martinez-Guryn K, Leone V, Chang EB. Regional diversity of the gastrointestinal microbiome. Cell Host Microbe 2019; 26(3): 314-324

11. Falony G, Joossens M, Vieira-Silva S, Wang J, Darzi Y, Faust K, Kurilshikov A, Bonder MJ, Valles-Colomer M, Vandeputte D, Tito RY, Chaffron S, Rymenans L, Verspecht C, De Sutter L, LimaMendez G, D'hoe K, Jonckheere K, Homola D, Garcia R, Tigchelaar EF, Eeckhaudt L, Fu J, Henckaerts L, Zhernakova A, Wijmenga C, Raes J. Population-level analysis of gut microbiome variation. Science 2016; 352(6285): 560-564

12. Abt MC, Osborne LC, Monticelli LA, Doering TA, Alenghat T, Sonnenberg GF, Paley MA, Antenus M, Williams KL, Erikson J, Wherry EJ, Artis D. Commensal bacteria calibrate the activation threshold of innate antiviral immunity. Immunity 2012; 37(1): $158-170$

13. Ganal SC, Sanos SL, Kallfass C, Oberle K, Johner C, Kirschning C, Lienenklaus S, Weiss S, Staeheli P, Aichele P, Diefenbach A. Priming of natural killer cells by nonmucosal mononuclear phagocytes requires instructive signals from commensal microbiota. Immunity 2012; 37(1): 171-186

14. Yeoh YK, Zuo T, Lui GC, Zhang F, Liu Q, Li AY, Chung AC, Cheung CP, Tso EY, Fung KS, Chan V, Ling L, Joynt G, Hui DS, Chow KM, Ng SSS, Li TC, Ng RW, Yip TC, Wong GL, Chan FK, Wong CK, Chan PK, Ng SC. Gut microbiota composition reflects disease severity and dysfunctional immune responses in patients with COVID-19. Gut 2021; 70(4): 698-706

15. Chen Y, Gu S, Chen Y, Lu H, Shi D, Guo J, Wu WR, Yang Y, Li Y, Xu KJ, Ding C, Luo R, Huang C, Yu L, Xu M, Yi P, Liu J, Tao JJ, 
Zhang H, Lv L, Wang B, Sheng J, Li L. Six-month follow-up of gut microbiota richness in patients with COVID-19. Gut 2022; 71(1): 222-225

16. Zuo T, Zhang F, Lui GCY, Yeoh YK, Li AYL, Zhan H, Wan Y, Chung ACK, Cheung CP, Chen N, Lai CKC, Chen Z, Tso EYK, Fung KSC, Chan V, Ling L, Joynt G, Hui DSC, Chan FKL, Chan $\mathrm{PKS}, \mathrm{Ng} \mathrm{SC}$. Alterations in gut microbiota of patients with COVID19 during time of hospitalization. Gastroenterology 2020; 159(3): 944-955.e8

17. Cao J, Wang C, Zhang Y, Lei G, Xu K, Zhao N, Lu J, Meng F, Yu L, Yan J, Bai C, Zhang S, Zhang N, Gong Y, Bi Y, Shi Y, Chen Z, Dai $\mathrm{L}$, Wang J, Yang P. Integrated gut virome and bacteriome dynamics in COVID-19 patients. Gut Microbes 2021; 13(1): 1-21

18. Bolger AM, Lohse M, Usadel B. Trimmomatic: a flexible trimmer for Illumina sequence data. Bioinformatics 2014; 30(15): 2114 2120

19. Langmead B, Salzberg SL. Fast gapped-read alignment with Bowtie 2. Nat Methods 2012; 9(4): 357-359

20. Wood DE, Lu J, Langmead B. Improved metagenomic analysis with Kraken 2. Genome Biol 2019; 20(1): 257

21. Robinson MD, McCarthy DJ, Smyth GK. edgeR: a Bioconductor package for differential expression analysis of digital gene expression data. Bioinformatics 2010; 26(1): 139-140

22. Hall M, Beiko RG. 16S rRNA gene analysis with QIIME2. Methods Mol Biol 2018; 1849: 113-129

23. Segata N, Izard J, Waldron L, Gevers D, Miropolsky L, Garrett WS, Huttenhower C. Metagenomic biomarker discovery and explanation. Genome Biol 2011; 12(6): R60

24. Love MI, Huber W, Anders S. Moderated estimation of fold change and dispersion for RNA-seq data with DESeq2. Genome Biol 2014; 15(12): 550

25. Beghini F, McIver LJ, Blanco-Míguez A, Dubois L, Asnicar F, Maharjan S, Mailyan A, Manghi P, Scholz M, Thomas AM, VallesColomer M, Weingart G, Zhang Y, Zolfo M, Huttenhower C, Franzosa EA, Segata N. Integrating taxonomic, functional, and strain-level profiling of diverse microbial communities with bioBakery 3. eLife 2021; 10: e65088

26. Newsome RC, Gauthier J, Hernandez MC, Abraham GE, Robinson TO, Williams HB, Sloan M, Owings A, Laird H, Christian T, Pride Y, Wilson KJ, Hasan M, Parker A, Senitko M, Glover SC, Gharaibeh RZ, Jobin C. The gut microbiome of COVID-19 recovered patients returns to uninfected status in a minoritydominated United States cohort. Gut Microbes 2021; 13(1): 1-15

27. Gu S, Chen Y, Wu Z, Chen Y, Gao H, Lv L, Guo F, Zhang X, Luo R, Huang C, Lu H, Zheng B, Zhang J, Yan R, Zhang H, Jiang H, Xu Q, Guo J, Gong Y, Tang L, Li L. Alterations of the gut microbiota in patients with coronavirus disease 2019 or H1N1 influenza. Clin Infect Dis 2020; 71(10): 2669-2678

28. Tao W, Zhang G, Wang X, Guo M, Zeng W, Xu Z, Cao D, Pan A, Wang Y, Zhang K, Ma X, Chen Z, Jin T, Liu L, Weng J, Zhu S. Analysis of the intestinal microbiota in COVID-19 patients and its correlation with the inflammatory factor IL-18. Med Microecol 2020; 5: 100023

29. Zuo T, Liu Q, Zhang F, Lui GC, Tso EY, Yeoh YK, Chen Z, Boon SS, Chan FK, Chan PK, Ng SC. Depicting SARS-CoV-2 faecal viral activity in association with gut microbiota composition in patients with COVID-19. Gut 2021; 70(2): 276-284
30. Tan Y, Liu F, Xu X, Ling Y, Huang W, Zhu Z, Guo M, Lin Y, Fu Z, Liang D, Zhang T, Fan J, Xu M, Lu H, Chen S. Durability of neutralizing antibodies and T-cell response post SARS-CoV-2 infection. Front Med 2020; 14(6): 746-751

31. Zhu C, Song K, Shen Z, Quan Y, Tan B, Luo W, Wu S, Tang K, Yang Z, Wang X. Roseburia intestinalis inhibits interleukin17 excretion and promotes regulatory $\mathrm{T}$ cells differentiation in colitis. Mol Med Rep 2018; 17(6): 7567-7574

32. Schirmer M, Smeekens SP, Vlamakis H, Jaeger M, Oosting M, Franzosa EA, Ter Horst R, Jansen T, Jacobs L, Bonder MJ, Kurilshikov A, Fu J, Joosten LAB, Zhernakova A, Huttenhower C, Wijmenga C, Netea MG, Xavier RJ. Linking the human gut microbiome to inflammatory cytokine production capacity. Cell 2016; 167(4): 1125-1136.e8

33. Naidoo CC, Nyawo GR, Sulaiman I, Wu BG, Turner CT, Bu K, Palmer Z, Li Y, Reeve BWP, Moodley S, Jackson JG, Limberis J, Diacon AH, van Helden PD, Clemente JC, Warren RM, Noursadeghi M, Segal LN, Theron G. Anaerobe-enriched gut microbiota predicts pro-inflammatory responses in pulmonary tuberculosis. EBioMedicine 2021; 67: 103374

34. Loomba R, Seguritan V, Li W, Long T, Klitgord N, Bhatt A, Dulai PS, Caussy C, Bettencourt R, Highlander SK, Jones MB, Sirlin CB, Schnabl B, Brinkac L, Schork N, Chen CH, Brenner DA, Biggs W, Yooseph S, Venter JC, Nelson KE. Gut microbiome-based metagenomic signature for non-invasive detection of advanced fibrosis in human nonalcoholic fatty liver disease. Cell Metab 2017; 25(5): 1054-1062.e5

35. Solé C, Guilly S, Da Silva K, Llopis M, Le-Chatelier E, Huelin P, Carol M, Moreira R, Fabrellas N, De Prada G, Napoleone L, Graupera I, Pose E, Juanola A, Borruel N, Berland M, Toapanta D, Casellas F, Guarner F, Doré J, Solà E, Ehrlich SD, Ginès P. Alterations in gut microbiome in cirrhosis as assessed by quantitative metagenomics: relationship with acute-on-chronic liver failure and prognosis. Gastroenterology 2021; 160(1): 206218.e13

36. Jie Z, Xia H, Zhong SL, Feng Q, Li S, Liang S, Zhong H, Liu Z, Gao Y, Zhao H, Zhang D, Su Z, Fang Z, Lan Z, Li J, Xiao L, Li J, Li R, Li X, Li F, Ren H, Huang Y, Peng Y, Li G, Wen B, Dong B, Chen JY, Geng QS, Zhang ZW, Yang H, Wang J, Wang J, Zhang X, Madsen L, Brix S, Ning G, Xu X, Liu X, Hou Y, Jia H, He K, Kristiansen $\mathrm{K}$. The gut microbiome in atherosclerotic cardiovascular disease. Nat Commun 2017; 8(1): 845

37. Depommier C, Everard A, Druart C, Plovier H, Van Hul M, VieiraSilva S, Falony G, Raes J, Maiter D, Delzenne NM, de Barsy M, Loumaye A, Hermans MP, Thissen JP, de Vos WM, Cani PD. Supplementation with Akkermansia muciniphila in overweight and obese human volunteers: a proof-of-concept exploratory study. Nat Med 2019; 25(7): 1096-1103

38. Rasmussen M, Johansson D, Söbirk SK, Mörgelin M, Shannon O. Clinical isolates of Enterococcus faecalis aggregate human platelets. Microbes Infect 2010; 12(4): 295-301

39. Ahmadrajabi R, Dalfardi MS, Farsinejad A, Saffari F. Distribution of Ebp pili among clinical and fecal isolates of Enterococcus faecalis and evaluation for human platelet activation. APMIS 2018; 126(4): 314-319

40. Lee W, Lim S, Son HH, Bae KS. Sonicated extract of Enterococcus faecalis induces irreversible cell cycle arrest in phytohemagglutinin- 
activated human lymphocytes. J Endod 2004; 30(4): 209-212

41. Nakamura A, Kurihara S, Takahashi D, Ohashi W, Nakamura Y, Kimura S, Onuki M, Kume A, Sasazawa Y, Furusawa Y, Obata Y, Fukuda S, Saiki S, Matsumoto M, Hase K. Symbiotic polyamine metabolism regulates epithelial proliferation and macrophage differentiation in the colon. Nat Commun 2021; 12(1): 2105

42. Proietti E, Rossini S, Grohmann U, Mondanelli G. Polyamines and kynurenines at the intersection of immune modulation. Trends Immunol 2020; 41(11): 1037-1050
43. Zhang M, Caragine T, Wang H, Cohen PS, Botchkina G, Soda K, Bianchi M, Ulrich P, Cerami A, Sherry B, Tracey KJ. Spermine inhibits proinflammatory cytokine synthesis in human mononuclear cells: a counterregulatory mechanism that restrains the immune response. J Exp Med 1997; 185(10): 1759-1768

44. Wang Q, Zhang M, Ding Y, Wang Q, Zhang W, Song P, Zou MH. Activation of NAD(P)H oxidase by tryptophan-derived 3-hydroxykynurenine accelerates endothelial apoptosis and dysfunction in vivo. Circ Res 2014; 114(3): 480-492 